\title{
Routine genetic screening with a multi-gene panel in patients with pheochromocytomas
}

\author{
Emilia Sbardella $\mathbb{D D}^{1,2} \cdot$ Treena Cranston ${ }^{3}$ Andrea M. Isidori ${ }^{2} \cdot$ Brian Shine $^{4}$. \\ Aparna Pal ${ }^{1} \cdot$ Bahram Jafar-Mohammadi $^{1} \cdot$ Greg Sadler $^{5} \cdot$ Radu Mihai $^{5}$. \\ Ashley B. Grossman ${ }^{1}$
}

Received: 11 February 2017 / Accepted: 19 April 2017

(C) Springer Science+Business Media New York 2017

\begin{abstract}
Purpose Several new gene mutations have been reported in recent years to be associated with a risk of familial pheochromocytoma. However, it is unclear as to whether extensive genetic testing is required in all patients.

Methods The clinical data of consecutive patients operated for pheochromocytoma over a decade in a tertiary referral center were reviewed. Genetic screening was performed using a 10-gene panel: RET, VHL, SDHB, SDHD, SDHA, SDHC, SDHAF2, MAX, TMEM127 and FH.

Results A total of 166 patients were analyzed: 87 of them had genetic screening performed (39 M: 44.8\%, $48 \mathrm{~F}$ : $55.2 \%$, age range $6-81$ years, mean $45 \pm 16.8$ years). In total, 22/87 (25.3\%) patients had germline mutations, while $65 / 87(74.7 \%)$ patients presented with apparently sporadic tumors. Germline VHL mutations were identified in $11.7 \%$ of patients, RET in $6.8 \%$ (five MEN2A/MEN2 and one MEN2B/MEN3), SDHD in 2.3\%, MAX in 2.3\%, SDHB in $1.1 \%$, and TMEM127 in $1.1 \%$ of patients. At diagnosis,
\end{abstract}

Emilia Sbardella

emi.sbardella@gmail.com

1 Department of Endocrinology, Oxford Centre for Diabetes, Endocrinology and Metabolism, Churchill Hospital, University of Oxford, Oxford, UK OX3 7LE, UK

2 Department of Experimental Medicine, Sapienza University of Rome, Viale Regina Elena, 324Rome 00161, Italy

3 Oxford Medical Genetics Laboratories, Churchill Hospital, University of Oxford, Oxford, UK OX3 7LE, UK

4 Department of Clinical Biochemistry,John Radcliffe Hospital, University of Oxford, Oxford, UK OX3 9DU, UK

5 Department of Endocrine Surgery, Churchill Hospital, Oxford University Hospitals NHS Foundation Trust, Oxford, UK OX3 7LE, UK
$15.1 \%$ of patients with unilateral non-syndromic pheochromocytoma showed germline mutations. We identified $19.7 \%$ of mutations in patients with unilateral-non-recurrent pheochromocytomas within 5 years vs. $50 \%$ in the recurrent-bilateral-metastatic group $(p=0.01)$. Germline mutations were more frequently seen with bilateral pheochromocytomas $(p=0.001)$ : $80 \%$ of patients with bilateral disease had germline mutations (4 VHL, 3 RET, 1 MAX). Conclusions The advent of rapid genetic screening using a gene-panel makes it feasible to screen large cohorts of patients and provides a valuable tool to contribute to the prediction of bilateral and malignant disease and to screen family members.

Keywords Pheochromocytoma $\cdot$ Gene $\cdot$ Genetic screening • Adrenal $\cdot$ Sporadic

$\begin{array}{ll}\text { Abbreviations } \\ \text { PHEO } & \text { Pheochromocytomas } \\ \text { PGL } & \text { Paraganglioma } \\ \text { NET } & \text { Neuroendocrine tumor } \\ \text { NMA } & \text { Normetanephrines } \\ \text { MA } & \text { Metanephrines } \\ \text { 3MT } & \text { 3-methoxythyramine } \\ \text { NGS } & \text { Next-generationsequencing }\end{array}$

\section{Introduction}

Pheochromocytoma (PHEO) is a rare neuroendocrine tumor (NET) arising from the adrenal chromaffin cells of the embryonic neural crest. Extra-adrenal PHEOs are referred to as paragangliomas (PGLs). PHEOs have an annual 
incidence of $1-4 / 10^{6}$ population [1], and are a significant albeit rare cause of hypertension, accounting for approximately 0.1 to $1 \%$ of patients with hypertension [1-4]. Despite their low frequency, PHEOs represent a significant challenge to clinicians as they can be lethal if untreated but can be subject to long-term cure, in the majority of cases, if diagnosed at an early stage and treated surgically.

The clinical presentation of the disease shows huge variability, with frequently non-specific symptoms and often mimicking other clinical conditions. The symptoms are usually secondary to the effects of high circulating catecholamines: hormonal hypersecretion can lead to hypertension, headaches, palpitations, diaphoresis, pallor, anxiety, and panic attacks [5].

Previously, PHEOs were thought to be associated with germline mutations in less than $10 \%$ of cases, the majority of these presenting with clear syndromic features. More recently it has been shown that up to $40 \%$ of PHEO are associated with an inherited mutation [6-9]. These are particularly present in younger patients, and those with multiple tumors [10], but may occur in patients with apparently simple sporadic tumors with no other syndromic features and no family history [11]. Currently, many different PHEO susceptibility genes have been reported: $N F 1$ [12, 13], RET [13], VHL [14], SDHD [15], SDHC [16], SDHB [17], EGLN1/PHD2 [18, 19], KIF1 [19], SDH5/ SDHAF2 [20], IDH1 [21], TMEM127 [22], SDHA [23], $M A X$ [24], FH and HIF2 [25], with new ones being added annually. On this basis, contemporary guidelines suggest that in the management of a patient with a PHEO, it is essential to consider genetic testing because of the high frequency of PHEOs associated with germline mutations [26].This is based on the high frequency of recurrent and malignant disease with some mutations, especially in $S D H B$ [26-28], and because diagnosing a hereditary syndrome in the proband could lead to earlier diagnosis and treatment of PHEOs, and other syndromic manifestations in relatives [28-30]. Nevertheless, current recommendations that genetic testing should be considered in each patient do not imply that genetic testing should be performed in all patients. Indeed, genetic testing has been considered to have limited incremental value in patients with no family history, no syndromic or malignant features, and with unilateral disease. The importance of the diagnosis of an inherited disease for at-risk families must be balanced against any negative impacts and the financial costs of genetic testing. Thus, recent guidelines recommend the use of a diagnostic algorithm driven by clinical aspects to evaluate the priorities for specific sequential genetic testing in PHEO patients with suspected germline mutations, proposing a selection of genes to be tested prioritized according to a syndromic or metastatic presentation [29]. Young age at presentation, positive family history, and the presence of multifocal
PHEO or bilateral adrenal tumors are also recognized as reasons for prioritizing patients for testing. Furthermore, tumor location and type of catecholamine secreted may also guide selection of suitable genes to test [29].

In this context, we utilized an alternative approach in which a 10-gene panel has been used to investigate the possibility of a germline syndrome in all patients presenting with PHEOs. The aim of this study was to evaluate whether such single extensive genetic testing is a valuable alternative approach to such patients. We have not included patients with PGLs as these are known to have a higher germline mutation rate.

\section{Methods}

\section{Patients}

Consecutive unselected patients operated in a tertiary referral centre between 2000 an 2015 were considered for inclusion in the study. Full genetic screening was performed in patients diagnosed after 2013 if this had not already been requested.

Patients operated before 2013 were invited for genetic screening if genetic testing had either not been performed or was incomplete. Of this subgroup, 65 patients who had not had any genetic screening performed at diagnosis or during early follow-up, recall for genetic testing was unsuccessful due to either inability to contact the patients, or the fact that they had moved region and the distance was too far to travel.

Moreover, of the 101 patients that had genetic testing, in 14 patients only a single gene was analyzed before the PHEO was diagnosed because of the typical clinical features of the suspected disease or for familial disease. Considering that in these patients genetic mutations were discovered before the diagnosis of a PHEO, we decided to exclude them from analysis (Fig. 1).

Therefore, 87 patients were screened, but for 8 there were syndromic reasons to identify a single gene, which then proved positive, while partial screening was only possible in a further 13 as the patients were unable to return for complete screening, which was thus only partial in these patients.

The diagnosis was based on elevated plasma and/or urinary metanephrines (MTs) and abnormal imaging, and then confirmed by histology. For more detailed evaluation of trends, changes, and outcomes, we divided our patients into two main subgroups: children (6-18 years) and adults (19 years and older). The adult group was again divided into two subcategories: (a) young adults (19-45 years), (b) older adults (over 45 years). 


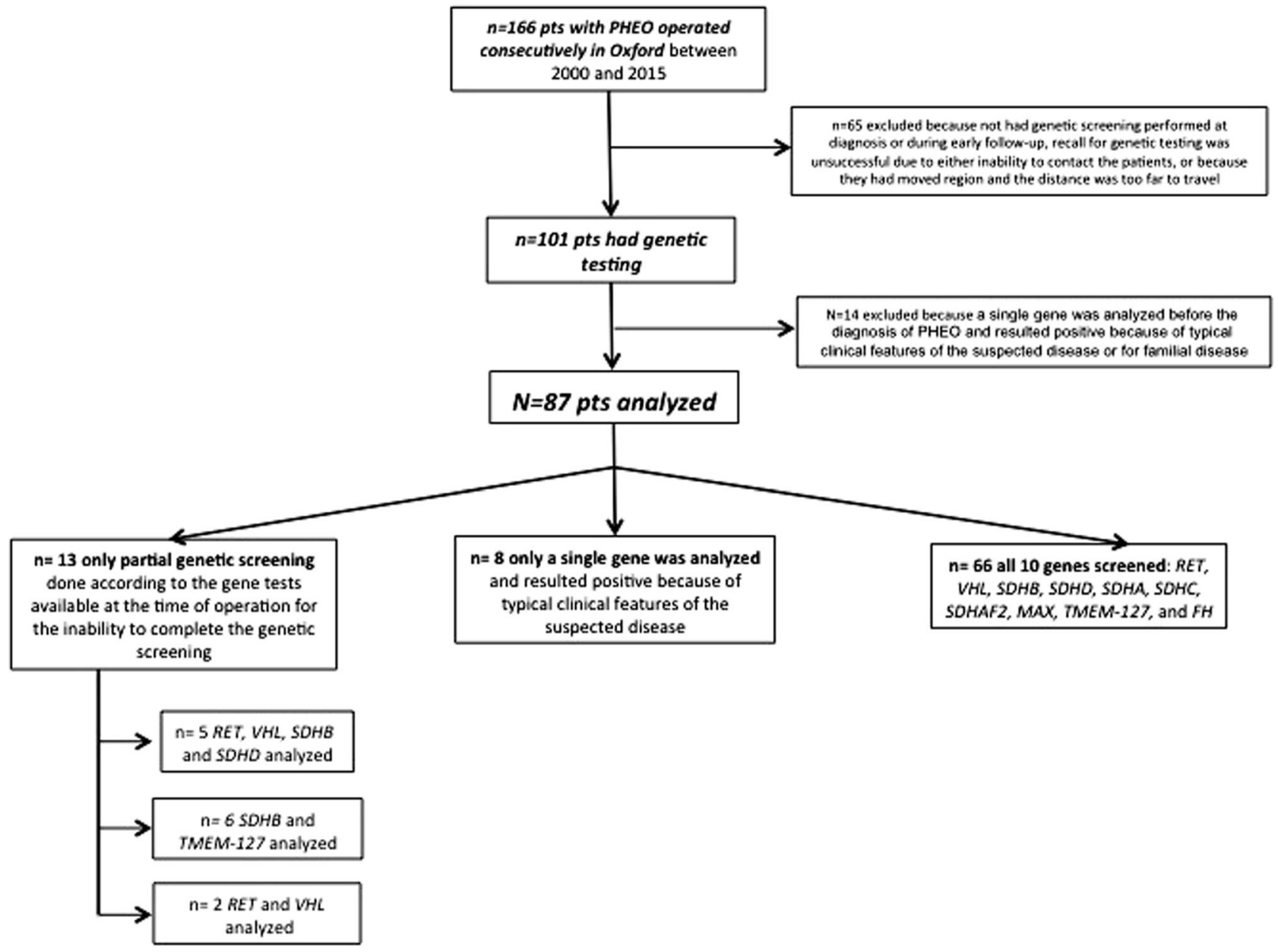

Fig. 1 Flow chart of requested genetic screening

\section{Genetics of PHEOs}

Genetic testing for mutations in principal PHEO susceptibility genes (RET, VHL, SDHB, SDHD, SDHA, SDHC, $S D H A F 2, M A X, T M E M 127$, and $F H$ ) was performed at the Oxford Medical Genetics Laboratories, Oxford University Hospitals NHS Trust, Churchill Hospital. NF1 was not tested as the diagnosis would have been made on clinical grounds.

Genomic DNA was extracted from peripheral blood leukocytes using standard procedures, and subjected to Sanger sequencing of the coding regions and exon-intron boundaries (+/-10 nt) of VHL, SDHB, SDHD, SDHA, $S D H C, S D H A F 2, M A X, T M E M 127$ and $F H$, plus targetted analysis of exons 10 and 11 of RET. Sanger sequencing was performed using the ABI BigDye 3.1 Terminator Cycle kit (Applied Biosystems), and analyzed using an ABI 3100 (Applied Biosystems) and mutation surveyor software (SoftGenetics). Additionally, analysis for deletions or duplications involving $S D H B, S D H D, S D H A F 2, S D H C$ and $V H L$ was undertaken by multiplex ligation-dependent probe amplification (MRC Holland kits P226-C1 and P016-C2).
In 13 patients, there was only partial genetic screening performed according to the gene tests available at the time of operation because of inability to contact the patients or because they were unable to attend the Department to complete the genetic screening because they had moved region and the distance was too far to travel.

\section{Biochemical testing and diagnosis}

The biochemical phenotype of the tumors was based on increased MTs [normetanephrine (NMT) or MT] in plasma and/or urine, as well as on plasma and/or urine 3methoxytyramine (3MT), when available. Twenty-fourhour urinary MTs, NMT, MT and 3MT were analyzed by liquid chromatography-mass spectrometry (LC-MS) and for 2013-2016 by LC-MS/MS: the reference values were identical. Urinary MTs measured showed an inter-assay imprecision of $6.58 \%$ at $0.56 \mu \mathrm{mol} / 24 \mathrm{~h}, 6.13 \%$ at 2.99 $\mu \mathrm{mol} / 24 \mathrm{~h}$ for normetadrenaline; $8.28 \%$ at $1.96 \mu \mathrm{mol} / 24 \mathrm{~h}$, $6.93 \%$ at $7.68 \mu \mathrm{mol} / 24 \mathrm{~h}$ for metadrenaline and of $11.27 \%$ 
at $0.51 \mu \mathrm{mol} / 24 \mathrm{~h}, 8.30 \%$ at $2.6 \mu \mathrm{mol} / 24 \mathrm{~h}$ for $3 \mathrm{MT}$. Plasma MTs were analyzed by LC-MS/MS.

The confirmation of the diagnosis of a metastatic PHEO was based on the clinical presentation and positive biochemistry/radiology or histopathological examination of tumors, and a previous history of PHEO. Positivity on PHEO-specific functional imaging studies further supported the diagnosis of metastatic PHEO, when available.

\section{Localization of the primary tumor and metastases}

Tumor presence was detected by CT and/or MRI of the abdomen or total-body CT in case of incidental findings. The majority of the patients (57/87: 65.5\%) underwent also

${ }^{123}$ I-MIBG scintigraphy to confirm a suspicious lesion and/ or to explore the presence of metastatic disease, and a very small number ${ }^{18}$ FDG-PET (5/87: 5.7\%).

\section{Follow-up}

Patients were usually followed-up initially every 6 months then yearly, unless other problems supervened, while metastatic patients were followed-up more frequently depending on clinical/biochemical/radiological evaluations followed by treatment recommendations and/or follow-up plans.

Of these patients, 61 underwent regular follow-up in our outpatient clinic with a minimum follow-up of 12 months and a maximum follow-up of 144 months.

\section{Statistical methods}

Data presented are expressed as means \pm standard deviations (SD) for continuous variables and as counts (\%) for categorical variables. For comparisons of single variables, $t$ tests were used while, for analyses involving multiple comparisons, the one-way analysis of variance or, for nonparametric data, the Mann-Whitney test, were used to determine statistical significance. Frequencies were compared using the $\chi^{2}$ test. $p<0.05$ was considered indicative of a statistically significant difference. Statistical analyses were performed using SPSS (version 17, Chicago, IL, USA).

The study was registered as an Audit Project by the Oxford University Hospitals Trust (Datix ID number 4061).

\section{Results}

\section{Characteristics of the study population}

Of the patients with PHEOs, 39/87 (44.8\%) were males and 48/87 (55.2\%) females. The mean age at diagnosis was 45 \pm 16.8 years (range: $6-81$ years): $72 / 87$ (82.8\%) underwent laparoscopic adrenalectomy, 12/87(13.8\%) open and 3/87 (3.4\%) laparoscopic-converted-to-open adrenalectomy.

Over a median follow-up of 50.4 months, there were 71/ 87 patients $(81.6 \%)$ with solitary tumors, $10 / 87$ (11.5\%) showed bilateral PHEO of which three were also recurrent, two were also metastatic and one both metastatic and recurrent; $8 / 87$ patients $(9.2 \%)$ had recurrent PHEO of which four were metastatic, three bilateral and one both metastatic and bilateral, and 9/87 patients (10.3\%) had metastatic tumors of which four were recurrent, two bilateral and one both recurrent and bilateral. Moreover, 9/87 showed another NET associated with PHEO, specifically five medullary thyroid carcinomas, three NETs of the pancreas and one bronchial carcinoid. In addition, 2/87 presented a non-NET tumor not clearly associated with known syndromes (one testicular tumor and one lymphoma).

Dividing by age subgroups : in those under 18 years of age 4/6 (66.6\%) had solitary tumors, 1/6 (16.7\%) had bilateral disease, 1/6 (16.7\%) had bilateral and recurrent, while none had metastatic disease. In the subgroup 18-45 years, $34 / 43(79.1 \%)$ had solitary tumors, two bilateral $(4.7 \%)$, one metastatic $(2.3 \%)$, two bilateral and recurrent $(4.7 \%)$, three with recurrence and metastases (7\%), and one bilateral and metastatic (2.3\%). In the subgroup of patients older than 45 years, $33 / 38(86.8 \%)$ had solitary tumors, one bilateral (2.6\%), one metastatic $(2.6 \%)$, one bilateral and metastatic (2.6\%), one with recurrence and metastases $(2.6 \%)$, and one bilateral, recurrent and metastatic $(2.6 \%)$.

Considering all patients younger than 45 years of age, $38 / 49$ (77.7\%) had solitary tumors, three bilateral $(6.1 \%)$, one metastatic (2\%), three bilateral and recurrent $(6.1 \%)$, three with recurrence and metastases $(6.1 \%)$, and one bilateral and metastatic (2\%).

\section{Characteristics of lesions at diagnosis/surgery}

At diagnosis/surgery of the primary tumor, three patients had metastatic disease, one patient was 33 years old and had liver and bone metastases, and showed a RET mutation; one patients was 41 years old and had a metastasis behind the vena cava, and his genetic screening for the panel of 10 genes analyzed was negative; the third was 36 years old, had bone metastases and his genetic screening for the panel of 10 genes analyzed was negative.

Four patients had bilateral disease at diagnosis/surgery: one patient was 25 years old and showed a VHL mutation, one patient was 33 years old, showed a RET mutation and had metastatic disease from the diagnosis, one patient was 36 years old and had an NF1 mutation, one patient was 56 years old and showed a MAX mutation. 


\section{Hereditary and apparently sporadic PHEO}

Overall, 87 patients underwent genetic screening, with 22/ $87(25.3 \%)$ patients demonstrating germline mutations diagnosed on the gene panel. Specifically, germline mutations were identified in $10(11.7 \%)$ patients for $V H L$ mutations, six (6.8\%) for RET (5 MEN2A/MEN2 and one MEN2B/MEN3), two (2.3\%) for SDHD, two (2.3\%) patients for $M A X$, and one each for $S D H B$ and for TMEM127. Moreover, in 6 patients NF1 was diagnosed based on clinical grounds (Fig. 2).

\section{Dividing by age subgroups}

In the group of pediatric patients $(<18$ years), $4 / 6(66.7 \%)$ had germline mutations; $15 / 43(34.9 \%)$ in the $18-45$ year subgroup and $3 / 38$ (7.9\%) in the older subgroup ( $>45$ years) ( $p=0.001$ comparing children vs. older adults and $p=0.003$ comparing young adults vs. older adults). The younger patients showed a significantly higher percentage of mutations compared to the older patients $(43.4 \%$ vs. $12.5 \%, p=$ 0.001 when comparing patients $<45$ to patients $\geq 45$ years old). In the pediatric subgroup, all the four patients with positive genetic screening showed a $V H L$ mutation. In the young adult subgroup (18-44 years), five subjects showed $V H L$ mutations, five RET mutation (four MEN 2A and one MEN 2B), two patients $S D H D$ mutations, one patient $S D H B$ mutation, one patient showed a $M A X$ mutation and one patient a TMEM127 mutation. In the older adults subgroup ( $\geq$ 45 years old), one subject showed a $V H L$ mutation, one a RET mutation compatible with MEN 2A and one patient showed a MAX mutation (Table 1 and Table 2).

\section{Dividing by new events of pheo}

Fourteen mutations were diagnosed in the 71 patients with unilateral-non-recurrent PHEOs (within 5 years) (19.7\%)

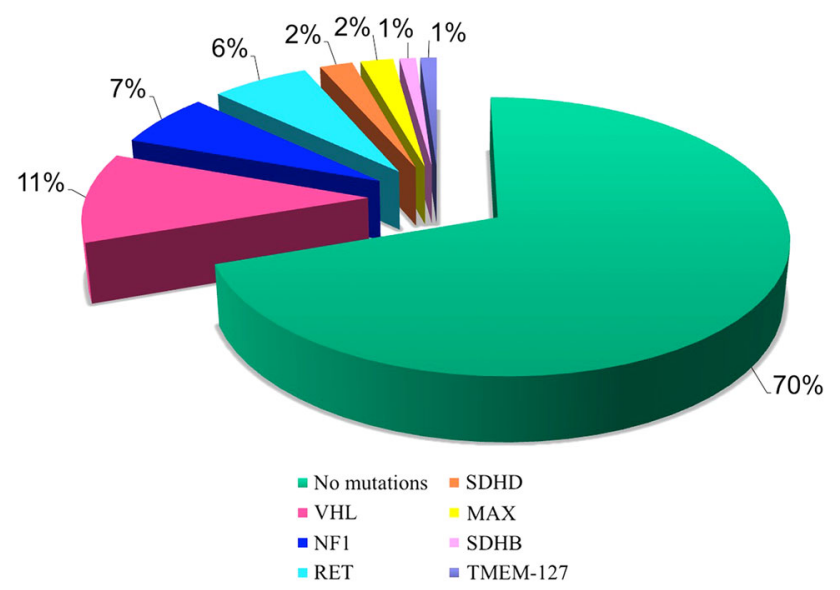

Fig. 2 Distribution of genetic mutations in the study population (included NF1 mutations diagnosed on clinical grounds)
Table 1 Germline mutation rates in different age subgroups

\begin{tabular}{lrccc}
\hline Mutation & $n$ & $<18$ years & $18-44$ years & $>45$ years \\
\hline MAX & 2 & $1 / 43(2.3 \%)$ & $1 / 38(2.6 \%)$ \\
RET & 6 & $5 / 43(11.6 \%)$ & $1 / 38(2.6 \%)$ \\
SDHB & 1 & $1 / 43(2.3 \%)$ & \\
SDHD & 2 & $2 / 43(4.6 \%)$ & \\
TMEM-127 & 1 & $1 / 43(2.3 \%)$ & \\
VHL & 10 & $4 / 6(66.7 \%)$ & $5 / 43(11.6 \%)$ & $1 / 38(2.6 \%)$ \\
No mutation & 65 & $2 / 6(33.3 \%)$ & $28 / 43(65.3 \%)$ & $35 / 38(92.2 \%)$ \\
identified & & & & \\
\hline
\end{tabular}

vs. $8 / 16(50 \%)$ in the recurrent-bilateral-metastatic group $(p=0.01)$.

Mutations were more frequently seen with bilateral $8 / 10$ (80\%) compared to unilateral PHEOs 14/77 (18.1\%) $(p=$ 0.001). In particular, in patients with bilateral disease the distribution of germline mutations were as follows: $4 \mathrm{VHL}$, 3 RET, 1 MAX.

\section{Dividing by the presence of other tumors associated with PHEOS}

Germline mutations were identified with a higher frequency in patients with PHEOs who also developed other NETs excluding PGLs compared to patients without evidence of NETs during the follow-up period (7/8:87.5\% vs. 15/ 79:19\% $p=0.001$ ). As a retrospective survey, follow-up was not standardised but was decided by individual clinicians: however, this was usually every 3-6 months, with a maximum time of 12 months.

No differences were observed when we compared patients with PHEOs associated with a tumor of neuroendocrine origin and patients with a tumor not of neuroendocrine origin.

\section{Age-related characteristics of primary and metastatic PHEOS}

The overall mean age of patients at the time of the diagnosis of the primary tumor was $45 \pm 16.8$ years (range 6-81 years). The mean age at initial diagnosis was significantly different when grouped by germline mutation. Patients with any identified germline mutation were younger at diagnosis than those without an identified mutation $(32.9 \pm 16.2$ years vs. $49 \pm 15.1$ years $p=0.001$ ).

In the recurrent-bilateral-metastatic group, comparing patients with genetic mutations and without genetic mutations, patients were significantly younger at the time of diagnosis of the primary tumor in the first group $(27.9 \pm 14.3$ years vs. $49.8 \pm 13.2$ years $p=0.007)$. The mean time interval to appearance of metastases was 
Table 2 Age at diagnosis and sex distribution for different mutation groups within PHEO patients

\begin{tabular}{llll}
\hline Mutation & $n$ & $\begin{array}{l}\text { Age at diagnosis, mean } \pm \text { SD, (min- } \\
\max )\end{array}$ & Sex M $n(\%), \mathrm{F} n(\%)$ \\
\hline MAX & 2 & $50 \pm 8.5(44-56)$ & F 2/2 (100\%) \\
RET & 6 & $37.3 \pm 17.5(19-69)$ & M 2/6 (33.3\%) F 4/6 (66.7\%) \\
SDHB & 1 & 22 & M 1/1 100\% \\
SDHD & 2 & $39 \pm 4.2(36-42)$ & M 2/2 100\% \\
TMEM-127 & 1 & 45 & M $1 / 1100 \%$ \\
VHL & 10 & $26.7 \pm 16.3(6-55)$ & M 5/10 (50\%) F 5/10 (50\%) \\
No mutation & 65 & $49.1 \pm 15(18-81)$ & M 29/65 (44.6\%) F 36/65 (55.4\%) \\
identified & & \\
\hline
\end{tabular}

$M$ male, $F$ female
$24.2 \pm 49.3$ months (range 12-144 months) and the mean age at first metastases $46.5 \pm 10.9$ years (range 33-61 years).

\section{Sporadic PHEOs and asymptomatic patients at diagnosis}

Considering patients with sporadic PHEOs, with no associated syndrome and without any family history for PHEOs at the time of diagnosis of the disease, 11/73(15.1\%) showed genetic mutations: three RET (two MEN2A and one MEN2B), two $V H L$, two $M A X$, two $S D H D$, one $S D H B$ and one TMEM127.

Of interest is the fact that in our population 16/87 (18.3\%), patients were 'incidentally diagnosed' to have a PHEO, without any symptoms or signs typical of a PHEO and not in the context of any syndromic condition: two of these (2/16: $12.5 \%)$ showed genetic mutations, one $S D H B$ and one TMEM127.

\section{Discussion}

Genetic screening has previously been considered to be expensive and time-consuming, but there are important implications of having a genetic diagnosis in apparently sporadic PHEOs. According to the American Society of Clinical Oncology's general recommendations for genetic screening [31], all patients with a risk of at least $10 \%$ of carrying a genetic mutation should be offered genetic testing, especially when the results would aid in diagnosis or influence the management of the patient or family members at hereditary risk of cancer [32]. In the present study, we have confirmed that more than $25 \%$ of patients with PHEOs showed germline mutations [5, 33], and that the rate can be as high as $55 \%$ if diagnosed by 18 years of age, as previously reported [5]. At the time of diagnosis of the primary tumor, patients were significantly younger in the group with genetic mutations compared to the group without genetic mutations. Moreover, in the recurrent-bilateral-metastatic group, the age of patients at the time of the diagnosis of primary tumor was significantly lower in patients with genetic mutations compared to patients without mutations. These data suggest that many patients with PHEOs should have genetic testing, especially when diagnosed at a young age. Furthermore, overall, in our study $15.1 \%$ of patients with apparently sporadic PHEOs and no family history showed genetic mutations, with a broad mix of differing aetiologies in line with the prevalence reported in a recent meta-analysis [34]. This high rate, plus the fact that there were varying genetic mutations, supports the use of a gene panel as opposed to algorithmic targeting of single genes sequentially.

Interestingly, in this population a high proportion of patients $(18.3 \%)$ were 'incidentally diagnosed' to have a PHEO, without any symptoms or signs typical of a PHEO and not in the context of any syndromic condition, and $12.5 \%$ of them showed germline mutations. These results suggest that the risk of a germline mutation is also significant in this group of lesions that are usually considered more indolent, and that proper and prompt genetic screening is essential, allowing the appropriate management and leading to a more accurate prognosis.

In our population, $50 \%$ of germline mutations were identified in patients with recurrent-bilateral-metastatic disease compared to $19.7 \%$ in the unilateral-non-recurrent PHEOs, suggesting that having a germline mutation increases the risk to develop a more complex disease, such as a recurrent or bilateral or a metastatic PHEO. In these patients, genetic screening is of particular value because it allows the clinician to provide a more focused management and follow-up of such patients at high risk of further disease.

We currently use a 10-gene panel analyzed with Sanger sequencing, rather than individual reporting for each gene according to other clinical or biochemical features as this is relatively cheap, efficient and, in pure cost terms, little different to a single gene assessment. Furthermore, NGS 
(next-generation sequencing) has been recently validated increasing the detection of germline mutations in patients with $\mathrm{PHEO}$, reducing processing time, time taken to report a result to a clinician, and lowering the cost compared to the conventional Sanger sequencing [35]. The use of a wide genetic panel appears to be especially appropriate for PHEO as even when there is no specific indication of a familial condition, with a unilateral non-recurrent PHEO, the germline mutation rate was still markedly above $10 \%$. This rate is somewhat above that reported in a recent metaanalysis [34], but similar to a NGS study [36] and is in accord with new recommendations [37].

\section{Limitations}

The principal limitations of this study are the relatively small sample size, even though collected from a major center over a prolonged period, with the lack of a consistent group of patients with mutations that were not in the context of a syndromic condition. Moreover, one of the main limitations is the retrospective nature of data collection in the majority of patients and our own institutional referral bias that may lead to a potential positive selection bias that may over-estimate the prevalence rate of more aggressive PHEOs. Furthermore, the gene panel was expanded over time, and even though we attempted to retrospectively reanalyze for germline mutations for previously-operated patients, in some instances this was not logistically possible. However, we act as a major referral centre for PHEOs in the context of the availability of experienced laparoscopic surgery. It is therefore likely the high rate of germline mutations seen in patients with apparently sporadic tumors, especially in younger patients and in the absence of a family history, is probably broadly representative of patients presenting to any major specialist unit.

\section{Conclusions}

While rare, PHEOs are more commonly associated with germline mutations than any other solid tumor type. Due to the advent of rapid genetic screening for a wide gene panel, more patients and families are being identified as mutation carriers and require lifelong screening for these potentially life-threatening tumors. The identification of a germline mutation in a patient with an apparent sporadic PHEO could lead to the early diagnosis of multiple or more aggressive tumors or additional syndromic neoplasms in the patient, as well as in relatives at risk. Knowledge of the heritable mutation has important implications for surveillance and monitoring of probands and their family members, and we believe multi-gene screening should become part of routine care for patients with PHEOs, especially as the costs of such screening fall.

\section{Compliance with ethical standards}

Conflict of interest The authors declare that they have no competing interests.

Ethical approval All procedures performed in studies involving human participants were in accordance with the ethical standards of the institutional and/or national research committee and with the 1964 Helsinki declaration, and its later amendments or comparable ethical standards.

\section{References}

1. J.T. Adler et al., Pheochromocytoma: current approaches and future directions. Oncologist 13(7), 779-793 (2008)

2. I. Ilias, K. Pacak, A clinical overview of pheochromocytomas/ paragangliomas and carcinoid tumors. Nucl. Med. Biol. 35(Suppl 1), S27-S34 (2008)

3. G. Eisenhofer et al., Pheochromocytoma catecholamine phenotypes and prediction of tumor size and location by use of plasma free metanephrines. Clin. Chem. 51(4), 735-744 (2005)

4. M.M. Walther, H.R. Keiser, W.M. Linehan, Pheochromocytoma: evaluation, diagnosis, and treatment. World J. Urol. 17(1), 35-39 (1999)

5. L. Fishbein, K.L. Nathanson, Pheochromocytoma and paraganglioma: understanding the complexities of the genetic background. Cancer Genet. 205(1-2), 1-11 (2012)

6. V.L. Martucci, K. Pacak, Pheochromocytoma and paraganglioma: diagnosis, genetics, management, and treatment. Curr. Probl. Cancer 38(1), 7-41 (2014)

7. L. Amar et al., Genetic testing in pheochromocytoma or functional paraganglioma. J. Clin. Oncol. 23(34), 8812-8818 (2005)

8. N. Burnichon et al., The succinate dehydrogenase genetic testing in a large prospective series of patients with paragangliomas. J. Clin. Endocrinol. Metab. 94(8), 2817-2827 (2009)

9. K. Pacak, S.J. Wimalawansa, Pheochromocytoma and paraganglioma. Endocr. Pract. 21(4), 406-412 (2015)

10. H.P. Neumann et al., Germ-line mutations in nonsyndromic pheochromocytoma. N. Engl. J. Med. 346(19), 1459-1466 (2002)

11. C.H. Lee et al., Genetics of apparently sporadic pheochromocytoma and paraganglioma in a Chinese population. Horm. Metab. Res. 47(11), 833-838 (2015)

12. D. Viskochil et al., Deletions and a translocation interrupt a cloned gene at the neurofibromatosis type 1 locus. Cell 62(1), 187-192 (1990)

13. L.M. Mulligan et al., Germ-line mutations of the RET protooncogene in multiple endocrine neoplasia type 2A. Nature 363 (6428), 458-460 (1993)

14. F. Latif et al., Identification of the von Hippel-Lindau disease tumor suppressor gene. Science 260(5112), 1317-1320 (1993)

15. R.E. Ferrell et al., Hereditary lymphedema: evidence for linkage and genetic heterogeneity. Hum. Mol. Genet. 7(13), 2073-2078 (1998)

16. S. Niemann, U. Muller, Mutations in SDHC cause autosomal dominant paraganglioma, type 3. Nat. Genet. 26(3), 268-270 (2000)

17. D. Astuti et al., Gene mutations in the succinate dehydrogenase subunit SDHB cause susceptibility to familial pheochromocytoma and to familial paraganglioma. Am. J. Hum. Genet. 69(1), 49-54 (2001) 
18. S. Lee et al., Neuronal apoptosis linked to EglN3 prolyl hydroxylase and familial pheochromocytoma genes: developmental culling and cancer. Cancer Cell. 8(2), 155-167 (2005)

19. S. Schlisio et al., The kinesin KIF1Bbeta acts downstream from EglN3 to induce apoptosis and is a potential 1p36 tumor suppressor. Genes Dev. 22(7), 884-893 (2008)

20. H.X. Hao et al., SDH5, a gene required for flavination of succinate dehydrogenase, is mutated in paraganglioma. Science 325(5944), 1139-1142 (2009)

21. J. Gaal et al., Isocitrate dehydrogenase mutations are rare in pheochromocytomas and paragangliomas. J. Clin. Endocrinol. Metab. 95(3), 1274-1278 (2010)

22. Y. Qin et al., Germline mutations in TMEM127 confer susceptibility to pheochromocytoma. Nat. Genet. 42(3), 229-233 (2010)

23. N. Burnichon et al., SDHA is a tumor suppressor gene causing paraganglioma. Hum. Mol. Genet. 19(15), 3011-3020 (2010)

24. I. Comino-Mendez et al., Exome sequencing identifies MAX mutations as a cause of hereditary pheochromocytoma. Nat. Genet. 43(7), 663-667 (2011)

25. C. Yang et al., Somatic mosaicism of EPAS1 mutations in the syndrome of paraganglioma and somatostatinoma associated with polycythemia. Hum. Genome. Var. 2, 15053 (2015)

26. A. Buffet et al., A decade (2001-2010) of genetic testing for pheochromocytoma and paraganglioma. Horm. Metab. Res. 44(5), 359-366 (2012)

27. F.M. Brouwers et al., High frequency of SDHB germline mutations in patients with malignant catecholamine-producing paragangliomas: implications for genetic testing. J. Clin. Endocrinol. Metab. 91(11), 4505-4509 (2006)

28. D.E. Benn et al., Clinical presentation and penetrance of pheochromocytoma/paraganglioma syndromes. J. Clin. Endocrinol. Metab. 91(3), 827-836 (2006)

29. J.W. Lenders et al., Pheochromocytoma and paraganglioma: an endocrine society clinical practice guideline. J. Clin. Endocrinol. Metab. 99(6), 1915-1942 (2014)
30. P.F. Plouin et al., European Society of Endocrinology Clinical Practice Guideline for long-term follow-up of patients operated on for a phaeochromocytoma or a paraganglioma. Eur. J. Endocrinol. 174(5), G1-G10 (2016)

31. M.E. Robson et al., American Society of Clinical Oncology policy statement update: genetic and genomic testing for cancer susceptibility. J. Clin. Oncol. 28(5), 893-901 (2010)

32. R. Martins, M.J. Bugalho, Paragangliomas/Pheochromocytomas: clinically oriented genetic testing. Int. J. Endocrinol. 2014, 794187 (2014)

33. L. Fishbein et al., Inherited mutations in pheochromocytoma and paraganglioma: why all patients should be offered genetic testing. Ann. Surg. Oncol. 20(5), 1444-1450 (2013)

34. J.P. Brito et al., Testing for germline mutations in sporadic pheochromocytoma/paraganglioma: a systematic review. Clin. Endocrinol. 82(3), 338-345 (2015)

35. E. Rattenberry et al., A comprehensive next generation sequencing-based genetic testing strategy to improve diagnosis of inherited pheochromocytoma and paraganglioma. J. Clin. Endocrinol. Metab. 98(7), E1248-E1256 (2013)

36. NGS in PPGL Study Group, R.A. Toledo, N. Burnichon, A. Cascon, D.E. Benn, J.P. Bayley, J. Welander, C.M. Tops, H. Firth, T. Dwight, T. Ercolino, M. Mannelli, G. Opocher, R. CliftonBligh, O. Gimm, E.R. Maher, M. Robledo, A.P. GimenezRoqueplo, P.L. Dahia, Consensus statement on next-generationsequencing-based diagnostic testing of hereditary phaeochromocytomas and paragangliomas. Nat. Rev. Endocrinol. 13(4), 233-247. doi:10.1038/nrendo.2016.185

37. M. Curras-Freixes et al., Recommendations for somatic and germline genetic testing of single pheochromocytoma and paraganglioma based on findings from a series of 329 patients. J. Med. Genet. 52(10), 647-656 (2015) 